\title{
RECESSION CONES OF NONCONVEX SETS AND INCREASING FUNCTIONS
}

\author{
GERALD BEER
}

\begin{abstract}
In this article a local characterization theorem is given for closed sets in a linear topological space that have recession cones with nonempty interior. This theorem is then used to characterize the class of upper semicontinuous increasing functions defined on closed $E_{+}^{d}$-recessional subsets of $E^{d}$.
\end{abstract}

1. Introduction. Let $S$ be a closed set in a linear topological space $L$ and let $x$ be a fixed point in $S$. A vector $y$ is called a direction of recession in $S$ at $x$ if for each positive $\lambda$ the vector $x+\lambda y$ is in $S$. If $S$ is convex, then the directions of recession are independent of $x$ and form a closed convex cone called the recession cone of $S$. The notion of recession cone has been used to sharpen many of the classical results of convexity theory. For example, the following version of Helly's Theorem holds [3]:

Let $\left\{C_{i}: i \in I\right\}$ be a collection of nonempty closed convex sets in d-dimensional Euclidean space. If the sets have no nontrivial common direction of recession and if each subcollection of at most $d+1$ sets has a nonempty intersection, then $\left\{C_{i}: i \in I\right\}$ has a nonempty intersection.

If $S$ is nonconvex, then the directions of recession in $S$ at a fixed point do not necessarily form a convex cone; furthermore, the directions of recession at one point do not determine the directions of recession at different points. However, it is easy to see that the common directions of recession do determine a closed convex cone. When can we conclude that a particular closed convex cone of recession at a fixed point of $S$ is shared by the other points of $S$ ? It is the purpose of this paper to answer this question. Moreover, we use the theory that we develop to characterize an important class of functions in mathematical economics.

Before proceeding, we set forth some terminology. If $S$ is a subset of a linear space $L$, then conv $S$ and cone $S$ will denote the convex hull and the cone generated by $S$, respectively. If $a$ and $b$ are two vectors in $L$, then $\operatorname{seg}[a, b]$ will denote the line segment joining $a$ and $b$, whereas $\operatorname{seg}(a, b)$ will mean the line segment exclusive of the endpoints. We say that " $a$ sees $b$ via $S$ " whenever $\operatorname{seg}[a, b] \subset S$. A real valued function $f$ is concave on $\operatorname{seg}[a, b]$ if

Presented to the Society, August 9, 1978; received by the editors December 22, 1977.

AMS (MOS) subject classifications (1970). Primary 52A05; Secondary 52A30, 46A99.

() 1979 American Mathematical Society 0002-9939/79/0000-0067/\$02.25 
whenever $\operatorname{seg}[x, y] \subset \operatorname{seg}[a, b]$ and $0<\lambda<1$, then

$$
f(\lambda x+(1-\lambda) y) \geqslant \lambda f(x)+(1-\lambda) f(y) \text {. }
$$

On the other hand, $f$ is called quasi-concave on $\operatorname{seg}[a, b]$ if $f$ restricted to each closed subinterval has an absolute minimum at an endpoint. Ordinary $d$ dimensional Euclidean space is symbolized by $E^{d}$. Finally, if $x \in E^{d}$, then $B_{\delta}(x)$ is the open $\delta$-ball in $E^{d}$ with center $x$.

\section{A local characterization theorem.}

Definition. Let $C$ be a closed convex cone in a linear topological space $L$. A set $S$ in $L$ is called $C$-recessional if $x+C \subset S$ whenever $x \in S$.

We shall show that a certain local property forces a convex cone of recession at a fixed point of a closed connected set $S$ in $L$ to serve as directions of recession at each point of $S$.

Definition. Let $C$ be a closed convex cone in $L$. A set $S$ in $L$ is called locally $C$-recessional if for each $x$ in $S$ there exists a neighborhood $N$ of $x$ such that whenever $y \in N \cap S$ and $z \in N \cap S$ and either $z \in y+C$ or $y \in z+C$, then $\operatorname{seg}[y, z] \subset S$.

THEOREM. Let $C$ be a closed convex cone with nonempty interior in a linear topological space L. If $S$ is a closed connected subset of $L$ that is locally $C$-recessional and contains some translate of $C$, then $S$ is $C$-recessional.

Proof. We use Zorn's Lemma. Let $\theta=\{D: S \supset D$ and $D$ is $C$ recessional $\}$. Since $S$ contains a translate of $C, \theta$ has a member other than the empty set. Clearly, $\theta$ is closed under unions of chains; so, $\theta$ has a maximal member, $S^{*}$, which must be closed. If $S^{*} \neq S$, the connectedness of $S$ forces the existence of a point $x$ in $S^{*}$, each neighborhood of which contains a point of $S \backslash S^{*}$. Let $p$ be an interior point of $C$, and let $N^{\prime}$ be a locally $C$-recessional neighborhood of $x$. By the continuity of

$$
(z, \alpha) \rightarrow \alpha(z+x)+(1-\alpha) x
$$

at $(z, \alpha)=(p, 0)$, for some positive $\alpha$ close to zero we can find an open set $E \subset C$ containing $p$ such that $\alpha(E+x)+(1-\alpha) x \subset N^{\prime}$. Since

$$
z \rightarrow \alpha(p+z)+(1-\alpha) z
$$

is continuous at $z=x$, there exists a neighborhood $N$ of $x$ in $N^{\prime}$ such that

$$
\alpha(p+N)+(1-\alpha) N \subset \alpha(E+x)+(1-\alpha) x \subset S^{*} \text {. }
$$

Now for each $q$ in $N \cap S$ the line segment joining $q$ to $\alpha(p+q)+(1-\alpha) q$ must lie in $S$ because of the local $C$-recessionality. However, since $\alpha(p+q)$ $+(1-\alpha) q$ is in $S^{*}$, each point on the ray emanating from $q$ through $\alpha(p+q)+(1-\alpha) q$ lies in $S$, so that $p$ is a direction of recession at each point $q$ in $N \cap S$.

We now show that each point $q$ of $N \cap S$ sees each point of int $(q+C)$ via $S$. Since each point of $q+C$ is a limit of interior points, we will be able to 
say that $q+C \subset S$ for each $q$ in $N \cap S$. Since

$$
S^{*} \cup\{C+q: q \in N \cap S\}
$$

contains $S^{*}$ as a proper subset, our assumption that $S \neq S^{*}$ has to be erroneous, and the proof will be complete. Let $p^{\prime}$ be an arbitrary point in int $C$ that is not a nonnegative multiple of $p$. First, we note that $p^{\prime}$ cannot be a negative multiple of $p$, or else the origin would be an interior point of $C$. It would then follow that $C=S=S^{*}=L$, and this contradicts our assumption that $S^{*}$ is a proper subset of $S$. Since $p^{\prime} \in$ int $C$ and $q+p+C$ $\subset S$, for all sufficiently large $\lambda$ the point $q+p$ sees $q+\lambda p^{\prime}$ via $S$. We need only show that $q$ sees $q+\lambda p^{\prime}$ via $S$. To establish this fact we use a technique developed by Klee [1].

Since $S$ is closed there is a point nearest to $q+\lambda p^{\prime}$ on $\operatorname{seg}\left[q+p, q+\lambda p^{\prime}\right]$ that $q$ sees via $S$. Denote this point by $a$. We shall show that $a=q+\lambda p^{\prime}$. If not, define $M$ as follows:

$$
\begin{aligned}
& M=\left\{y: y \in \operatorname{seg}[q, a] \text { and } \operatorname{conv}\left\{y^{\prime}, a, y\right\} \subset S\right. \\
& \left.\quad \text { for some } y^{\prime} \in \operatorname{seg}\left(a, q+\lambda p^{\prime}\right)\right\} .
\end{aligned}
$$

Clearly, $a \in M$; so, $M$ is nonempty. We claim that $M$ is both open and closed in $\operatorname{seg}[q, a]$. To see that $M$ is closed, fix $z$ in cl $M$ and let $N^{*}$ be a locally $C$-recessional neighborhood of $z$. If $\operatorname{seg}[z, q]$ contained points of $M$, then clearly $z$ is in $M$, too. Otherwise, we can find $y$ in $M \cap N^{*}$ where $y$ is between $z$ and $a$. By the definition of $M$ there exists $y^{\prime}$ in $\operatorname{seg}\left(a, q+\lambda p^{\prime}\right)$ such that $\operatorname{conv}\left(\left\{y^{\prime}, a, y\right\}\right) \subset S$. Since $a-z \in$ int $C$, we see that $N^{*} \cap(z+C)$ contains a nondegenerate subsegment $\operatorname{seg}\left[y, y^{*}\right]$ of $\operatorname{seg}\left[y, y^{\prime}\right]$. It follows that $\operatorname{conv}\left\{z, y, y^{*}\right\} \subset S$. Denoting the intersection of the ray emanating from $z$ through $y^{*}$ with $\operatorname{seg}\left(a, q+\lambda p^{\prime}\right)$ by $z^{\prime}$, we see that $\operatorname{conv}\left\{z^{\prime}, a, z\right\} \subset S$. Hence, $M$ is closed. To show that $M$ is open, we use the same geometrical technique, but since our notation is sufficiently cluttered, we leave this detail to the reader. We now know that $M$ is a nonempty open and closed subset of $\operatorname{seg}[q, a]$. Thus $M=\operatorname{seg}[q, a]$ so that $q$ sees a nondegenerate subsegment of $\operatorname{seg}\left[a, q+\lambda p^{\prime}\right]$ via $S$. Since this is not compatible with the definition of $a$, we conclude that $a=q+\lambda p^{\prime}$ and we are done.

Simple counterexamples in the plane show that none of the hypotheses of the theorem can be omitted. The condition of most interest is that we must give our cone of recession a nonempty interior. To see that this is essential, consider

$$
S=\left\{(x, y): x^{2}+y^{2} \leqslant 1 \text { or } y=0\right\} .
$$

Although $S$ is locally recessional with respect to the horizontal axis, $S$ is not recessional with respect to this cone. To see that $S$ must actually contain a translate of the cone in question, consider

$$
S=\{(x, y): x>0, y>0 \text { and either } x+y<1 \text { or } y=0\} .
$$


$S$ is locally recessional with respect to the first quadrant, but $S$ does not even contain a translate of this cone. The importance of the connectedness condition is just as simple to verify.

3. Recession cones and increasing functions. Shephard's modern axiomatic treatment of production functions [4] is just one of the many models in economic theory that involve upper semicontinuous functions defined on $E_{+}^{d}$, the set of all nonnegative vectors in $E^{d}$, that are increasing: if $\alpha_{i}>\beta_{i}$ for $i=1, \ldots, d$, then

$$
f\left(\alpha_{1}, \ldots, \alpha_{d}\right) \geqslant f\left(\beta_{1}, \ldots, \beta_{d}\right) .
$$

Upper semicontinuous increasing functions can be described in terms of recessionality properties: each level set $\{x: f(x) \geqslant \theta\}$ is closed and $E_{+}^{d}$ recessional. Actually, such a function can be described by a single less familiar condition: its hypograph $\{(x, \mu): \mu \leqslant f(x)\}$ is closed and recessional with respect to the cone generated by $\left\{e_{1}, \ldots, e_{d},-e_{d+1}\right\}$ where $\left\{e_{1}, \ldots, e_{d+1}\right\}$ is the standard basis in $E^{d+1}$. Our main theorem allows us to characterize such functions by the local behavior that they must exhibit.

THEOREM Let $f$ be an upper semicontinuous function defined on a closed set $S$ in $E^{d}$ that is $E_{+}^{d}$-recessional. Suppose that there exists $s$ in $S$ such that $f$ is bounded below on each ray in $s+E_{+}^{d}$ with initial point $s$. Suppose that for each $x$ in $S$ there exists $\delta>0$ such that whenever $u$ is a unit vector in $E_{+}^{d}$ and $f\left(x+\theta_{1} u\right) \leqslant f(x)+\delta$ and $f\left(x+\theta_{2} u\right) \leqslant f(x)+\delta$ where $-\delta<\theta_{1}<\theta_{2}<$ $\delta$, then $f$ behaves the following way on $\operatorname{seg}\left[x+\theta_{1} u, x+\theta_{2} u\right]$ :

(i) if $f\left(x+\theta_{2} u\right) \geqslant f\left(x+\theta_{1} u\right)$, then $f$ is quasi-concave.

(ii) if $f\left(x+\theta_{2} u\right)<f\left(x+\theta_{1} u\right)$, then $f$ is concave.

Then $f$ is an increasing function.

Proof. Let $y$ be a fixed vector in $S$ and let $u$ be a unit vector in $E_{+}^{d}$. We claim that if $f$ is bounded below on $\{y+\lambda u: \lambda \geqslant 0\}$, then $f$ is actually increasing on the ray. It suffices to show that the hypograph of $f$ restricted to this ray is $C$-recessional where $C=\operatorname{cone}\left\{u,-e_{d+1}\right\}$. Let $\rho$ be a lower bound for $f$ on the ray. The hypograph of $f$ restricted to the ray contains a translate of $C$, namely $(y, \rho)+C$. Since the ray is closed and $f$ is upper semicontinuous, the hypograph of $f$ restricted to the ray is closed. This subset of the hypograph of $f$ is also connected, for it is the union of vertical rays each of which intersects the connected set $(y, \rho)+C$. To conclude that $f$ is increasing on the ray, it remains only to show that the hypograph of $f$ restricted to the ray is locally $C$-recessional.

To this end fix $x$ on the ray and choose $\lambda \leqslant \delta$ so small that the local condition holds and $f$ is bounded above in $B_{\delta}(x) \cap S$ by $f(x)+\delta$. We show that if $\mu \leqslant f(x)$, then $N=\beta_{\lambda}(x) \times(-\infty, f(x)+\delta)$ is a $C$-recessional neighborhood of $(x, \mu)$ relative to the hypograph restricted to the ray. Choose $(y, \alpha)$ and $(z, \beta)$ in the hypograph of $f$ restricted to the ray and in $N$ where 
$z \geqslant y$ componentwise and $\beta \leqslant \alpha$. If $f(y) \leqslant f(z)$, then

$$
f(\lambda y+(1-\lambda) z) \geqslant f(y) \geqslant \alpha
$$

for each $\lambda$ in $(0,1)$. Hence $(\lambda y+(1-\lambda) z, \alpha)$ belongs to the hypograph for each $\lambda$ so that $\operatorname{seg}[(y, \alpha),(z, \beta)]$ is contained in the hypograph, too. On the other hand, if $f(y)>f(z)$, then

$$
f(\lambda y+(1-\lambda) z) \geqslant \lambda f(y)+(1-\lambda) f(z) \geqslant \lambda \alpha+(1-\lambda) \beta,
$$

and we again conclude that $\operatorname{seg}[(y, \alpha),(z, \beta)]$ is in the hypograph. Since $x$ was arbitrary, the hypograph is locally $C$-recessional and the claim is established.

Next we verify that $f$ must actually be bounded below on each ray of the form $\{y+\lambda u: \lambda \geqslant 0\}$ where $y \in S$ and $u \in$ int $E_{+}^{d}$. From the first part of the proof we know that $f(s)$ is a lower bound for $f$ restricted to $s+E_{+}^{d}$ because $f$ must be increasing on each ray in $s+E_{+}^{d}$ with initial point $s$. Evidently, there exists positive $\lambda_{0}$ such that $\left\{y+\lambda u: \lambda \geqslant \lambda_{0}\right\} \subset s+E_{+}^{d}$ whence $f(y+\lambda u) \geqslant f(s)$ on this portion of the ray. The function is also bounded below on $\operatorname{seg}\left[y, y+\lambda_{0} u\right]$, for if not we can find a sequence $\left\{r_{n}\right\}$ in the segment convergent to a point $r$ such that $f\left(r_{n}\right)<-n$. Since $f(r)>f\left(r_{n}\right)$ for all large $n$, it is clear that the local quasi-concavity condition is violated at $r$.

We have shown that $f(y+v) \geqslant f(y)$ for each $y$ in $S$ and each $v$ in int $E_{+}^{d}$. The upper semicontinuity of $f$ implies that $f(y+v) \geqslant f(y)$ for each $v$ in $E_{+}^{d}$, and the theorem is proved.

Some counterexamples are in order. First, note that local quasi-concavity in the "positive direction" alone will not suffice, for the function defined by

$$
f(x)= \begin{cases}x & \text { if } 0 \leqslant x \leqslant 1 \\ e^{1-x} & \text { if } x>1\end{cases}
$$

fails to be increasing. It is clear that we must require that $f$ be in some sense bounded below, for a decreasing linear functional satisfies the other hypotheses. Finally, the upper semicontinuity condition is essential, for

$$
f(x)= \begin{cases}(1-x)^{-1} & \text { if } 0 \leqslant x<1, \\ 0 & \text { if } x \geqslant 1,\end{cases}
$$

fails to be increasing. (The reader should check that the local condition is satisfied in a neighborhood of $x=1$.)

\section{REFERENCES}

1. V. L. Klee, Convex sets in linear spaces, Duke Math. J. 18 (1951), 443-466.

2. A. W. Roberts and D. E. Varberg, Convex functions, Academic Press, New York, 1973.

3. R. T. Rockafellar, Convex analysis, Princeton Univ. Press, Princeton, N.J., 1970.

4. R. W. Shephard, Theory of cost and production functions, Princeton Univ. Press, Princeton, N.J., 1970.

Department of Mathematics, California State University, los Angeles, California 90032 